\title{
Sindrome postlaminectomía lumbar II. Tratamiento del dolor mediante técnicas de neuromodulación
}

\author{
F.J. Robaina Padrón
}

Unidad del Dolor Crónico y Neurocirugía Funcional. Hospital Universitario de Gran Canaria Dr. Negrín. Las Palmas de Gran Canaria.

\section{Resumen}

La aplicación de las técnicas de neuromodulación avanza rápidamente en los distintos aspectos de la medicina actual. La neuromodulación se define como: "la realización de intervenciones terapéuticas mediante aparatos implantables para modificar la función del sistema nervioso central, periférico o autonómico". Tras la cirugía discal y vertebral en general, se pueden generar síndromes dolorosos persistentes tanto a nivel axial como distal en las extremidades inferiores. La situación actual de la cirugía de la columna vertebral degenerativa en relación al alivio del dolor lumbar crónico, por enfermedad degenerativa discal y estenosis de canal, empleando los métodos de la medicina basada en la evidencia, concluyen, que la vía instrumentista y fijadora no es precisamente la ideal después de $\mathbf{2 0}$ años de utilización de las mismas. Las técnicas de neuromodulación representan un escalón superior en el algoritmo de manejo de estos síndromes dolorosos, ya que permiten con mayor frecuencia solventar muchos casos de dolor persistente tras la cirugía sin la necesidad de realizar otra nueva cirugía. Se describen las técnicas de neuromodulación (estimulación medular e infusión espinal de medicamentos) aplicables al síndrome postlaminectomía lumbar y se detallan los aspectos técnicos para la realización de las mismas. Las técnicas de neuromodulación aplicadas al manejo del dolor postcirugía discal representan una alternativa válida en comparación con la nueva cirugía o la artrodesis. Los neurocirujanos estamos llamados de nuevo a participar activamente en el campo de la neuromodulación para el alivio del dolor crónico, el de los movimientos anormales, la psicocirugía y el restablecimiento de las funciones neurológicas perdidas.

PALABRAS CLAVE: Síndrome postlaminectomía lumbar. Alivio del dolor. Técnicas de neuromodulación. Estimulación eléctrica medular. Infusión espinal de medicamentos.

Recibido: 20-08-06. Aceptado: 10-09-07
Lumbar post-laminectomy syndrome: II. Pain management using neuro-modulation techniques

Summary

The application of neuro-modulation techniques in general is currently gaining acceptance in various aspects of medicine. Neuro-modulation is defined as: "Terapeutical interventions using implantable devices to modify the functioning of central, peripheral and and autonomic nervosus sysmtems". Following lumbar disc surgery, or lumbar spine surgery in general, several chronic pain syndromes can result, either in the lumbar region and/or in the lower limbs. The current status is for the application of surgery to the degenerative spine (degenerative disc disease and lumbar stenosis) for the relief of chronic pain. A review of the methodology of evidence based medicine, show that the instrumented and fusion techniques are not the answerd despite 20 years of the use of these techniques following failure of surgery for the relief of back pain syndrome. Neuromodulation techniques represent a step in the right direction for the management of these chronic pain syndromes. Frecuently they enable the resolution of chronic pain following spine surgery without having to resort to repeat surgery. We describe here the different neuro-modulation techniques (spinal cord stimulation, spinal drug infusions) which can be used in the case of back surgery failure, and we describe technical aspects and "tricks of the trade" for the correct implantation of the devices used in techniques. Neuro-modulation techniques are applied to the management of chronic pain following disc surgery and represent a valid alternative to repeat surgery and/or arthrodesis (instrumented or not). Neurosurgeosn are again called to play active roles in the field of neuro-modulation for the treatment

Abreviaturas. CRPS II: sindrome de dolor regional complejo tipo II. DBS: estimulación cerebral profunda. EMECP: estimulación medular epidural de los cordones posteriores. RNM: resonancia nuclear magnética. TAC: tomografía axial computarizada. 
of pain as well as in the field of neuro-modulation of abnormal movements disorders, for psychosurgery and the revovery of neurological functions.

KEY WORDS: Failed back surgery sindrome. Pain relief. Neuro-modulation techniques. Spinal cord stimulation. Spinal drug infusion.

\section{Introducción}

El campo de la neuromodulación es uno de los que más rápidamente avanza en los distintos aspectos de la medicina actual. La neuromodulación se define como: "la realización de intervenciones terapéuticas mediante aparatos implantables para modificar la función del sistema nervioso central, periférico o autonómico". Aparatos implantables como los estimuladores medulares se aplican en el manejo del dolor, la espasticidad y el restablecimiento de funciones gastrointestinales o urinarias. La estimulación cerebral profunda se aplica en el campo de los movimientos anormales, el dolor y algunas alteraciones psiquiátricas. La estimulación cortical se aplica en el dolor, la epilepsia y algunas alteraciones psiquiátricas. El empleo de bombas de infusión de analgésicos y sustancias antiespáticas se encuentra dentro del abanico de las técnicas de neuromodulación. La existencia de una publicación internacional que solo incluye artículos de neuromodulación, refleja el auge que este campo esta tomando en la medicina actual $^{10}$.

Tras la cirugía discal y vertebral en general, se pueden generar síndromes dolorosos persistentes tanto a nivel axial como distal en las extremidades inferiores. Los aspectos clínicos fundamentales de estos síndromes dolorosos y las diferentes posibilidades terapéuticas intervencionistas ya han sido descritas previamente ${ }^{17}$.

Los resultados de la cirugía de la hernia discal son bastante contradictorios, no existiendo en la literatura mundial ningún trabajo científico que compare la cirugía y el tratamiento conservador sin ningún tipo de sesgo científico en la recogida de datos ${ }^{24}$.

La utilización de nuevos tratamientos emergentes, percutáneos, en la cirugía de la hernia discal, hacen que las indicaciones quirúrgicas en la hernia discal lumbar deban tomarse con reservas en aquellos casos no paralizantes o de dolor incontrolable, debido a la existencia de métodos alternativos no quirúrgicos ${ }^{1,2,6,9,14,18}$.

Publicaciones recientes que revisan la situación actual de la cirugía de la columna vertebral degenerativa en relación al alivio del dolor lumbar crónico, por enfermedad degenerativa discal y estenosis de canal, empleando los métodos de la medicina basada en la evidencia, concluyen, que la vía instrumentista y fijadora no es precisamente la ideal después de 20 años de utilización de las mismas ${ }^{3,20}$. El empleo de tratamientos cognitivos y ejercicios de rehabilitación apropiados consiguen tasas similares de éxito en el control del dolor que la fusión espinal posterolateral instrumentada transpedicular, en pacientes con dolor lumbar persistente tras cirugía de hernia discal ${ }^{4,16,23}$.

Las técnicas de neuromodulación representan por lo tanto un escalón superior en el algoritmo de manejo de este tipo de síndromes dolorosos, dado que permiten con mayor frecuencia solventar muchos casos de dolor persistente tras la cirugía lumbar sin la necesidad de realizar nueva cirugía cuyo resultado, como ya se ha mencionado, es incierto.

La neuroestimulación transcutánea no es una técnica intervencionista pero se cita aquí como una técnica básica de neuromodulación aplicable en las etapas iniciales del manejo de este tipo de pacientes. La estimulación de nervios periféricos no es aplicable generalmente a este tipo de patología, ya que sus indicaciones más precisas son en los casos de dolor regional complejo tipo II (CRPS II- Causalgia), que no suelen presentarse tras este tipo de cirugía.

La estimulación medular epidural a nivel de los cordones posteriores(EMECP) entre D9-D12 consigue un importante alivio del dolor en la extremidad en un número importante de pacientes, siendo más variable el control del dolor en la zona lumbar.

Recientemente, los nuevos diseños de electrodos multipolares y sistemas de screening computarizados parece que permiten identificar por parte del paciente las mejores combinaciones de polaridad para obtener parestesias inducidas en la región lumbar y por lo tanto, conseguir alivio de la lumbalgia.

La estimulación cerebral profunda a nivel talámico o de la sustancia gris periventricular (DBS), es un procedimiento que no reporta beneficios en este tipo de pacientes, por lo que se practican excepcionalmente.

Destacar y recordar en este punto, que las técnicas de neuromodulación, son procedimientos reversibles, a diferencia de las técnicas neuroablativas clásicas, representando por lo tanto un adelanto terapéutico muy importante para el manejo del dolor crónico en general, sin miedo a las secuelas irreversibles que con alguna frecuencia se presentaban con las técnicas quirúrgicas.

\section{Técnicas de electroestimulación medular en el trata- miento del dolor vertebral}

La EMECP es una técnica ampliamente desarrollada en nuestro país, constituyendo una alternativa terapéutica de elección en muchos síndromes de dolor crónico no neoplásico en gran número de las Unidades del Dolor Crónico de España y/o algunos Servicios de Neurocirugía. La EMECP fue la repuesta clínica a la teoría de la puerta de entrada formulada por Melzak y Wall en 1965 ${ }^{13}$. Las 
Tabla I

\section{Lesiones originadas en los nervios periféricos y raíces nerviosas}

Neuropatías postraumáticas

- Heridas Traumáticas

- Cirugía

- Atrapamientos nerviosos

- Cicatrices

Distrofia simpática refleja (Síndrome de dolor regional complejo tipo I)

Causalgia ( Síndrome de dolor regional complejo tipo II )

Dolor postamputación ( dolor de muñón y miembro fantasma doloroso)

Neuropatía diabética

Lesiones parciales de plexos nerviosos (traumáticas, neoplásicas, postradioterapia)

Neuritis postherpética

Síndrome Postlaminectomía lumbar (lumbalgia y ciática postquirúrgica, aracnoiditis y fibrosis epidural)

\section{Lesiones de la médula espinal}

- Esclerosis múltiple

- Dolor en la paraplejia (dolor radicular a nivel lesional y dolor sublesional )

\section{Enfermedad Vascular periférica}

- Arteriosclerosis

- Diabetes Mellitus

- Enfermedad de Buerger

- Esclerodermia

- Enfermedad Idiopática de Raynaud

\section{Angina péctoris refractartia con dolor incontralable}

posibilidades terapéuticas de la EMECP son muy amplias, siendo aplicable en multitud de síndromes dolorosos. En la Tabla I, se muestran los síndromes dolorosos capaces de responder a la EMECP. Los porcentajes de alivio del dolor de la lumbalgia y ciática oscilan alrededor de un 50-60\% de resultados favorables tras varios años de seguimiento ${ }^{19}$.

Estudios a doble ciego han demostrado que si se descartan inestabilidades postquirúrgicas y compresiones radiculares y si han fracasado los tratamientos conservadores médicos (farmacológicos) y rehabilitadores, el control de la ciática persistente de origen neuropático es mucho más eficaz mediante neuroestimulación medular que mediante reintervención quirúrgica. La nueva cirugía, en muchas ocasiones, origina un empeoramiento de la sintomatología previa. No obstante, en algunas situaciones, la exploración clínica y los estudios neuro-radiológicos pueden sugerir la necesidad de reintervenir quirúrgicamente. La presencia de neurocirujanos y/o cirujanos de columna en el equipo multidisciplinar de valoración de estos problemas es imprescindible ${ }^{19}$.

Estudios costo-beneficio más recientes demuestran que a los 5 años el ahorro económico de los pacientes implantados con estimuladores medulares es significativamente mejor en comparación con los no implantados, incluso un $15 \%$ de los pacientes implantados volvió a su trabajo habitual por ninguno de los no implantados ${ }^{12}$. 
Un estudio reciente realizado por North ${ }^{15}$ en la Universidad de Baltimore USA, analizando todos los casos publicados entre 1967 y 2002 demuestra la eficacia de esta técnica a largo plazo para el control del dolor en la lumbalgia y la ciática postquirúrgica. Algunas publicaciones revisadas por el mismo autor, que comparan diferentes artículos recientes sobre la materia, demuestran que se obtienen mejores resultados en el control del dolor postcirugía discal mediante neuroestimulación medular que con la nueva cirugía. No obstante, el propio autor hace mención a la necesidad de nuevos estudios comparativos que puedan definir correctamente cual de las dos técnicas es más efectiva en igualdad de mecanismo fisiopatológico y del tipo de dolor que se vaya a tratar.

\section{Técnica de implante de electrodos y estimuladores medulares}

La técnica de neuroestimulación medular se realiza en quirófano, con la máxima esterilidad, como cualquier intervención quirúrgica de neurocirugía espinal. El paciente es colocado en decúbito prono con un cojín bajo el abdomen de forma que se neutralice la lordosis fisiológica lumbar y se acentúe ligeramente la cifosis dorsal lo que facilita la progresión del electrodo en el espacio epidural posterior. Se puede utilizar algún tipo de sedación intravenosa, pero en todo momento el paciente debe estar completamente alerta ya que debe indicar la percepción de las parestesias inducidas sobre la zona del dolor.

En una primera fase, con la aguja de Thuoy modificada que viene con el set de electrodos del sistema de estimulación que vayamos a emplear, mediante la técnica de pérdida de resistencia o de la gota pendiente, se localiza el espacio epidural posterior a nivel L1-L2, intentando acceder al mismo en la línea media, tras una punción de la piel paramedial derecha o izquierda en un nivel inferior lo que garantiza la oblicuidad de la aguja al acceder al espacio epidural. Posteriormente, se introduce el electrodo haciéndolo progresar cranealmente hasta alcanzar el nivel vertebral D9-D10. El electrodo puede ser tetrapolar u octopolar, localizado en la línea media, o pueden implantarse dos electrodos tetrapolares en paralelo, lo que implica una doble punción. A mayor número de puntos activos mayor posibilidad de crear un mejor campo eléctrico. Si el dolor predomina en un lado el electrodo puede desviarse hacia el mismo, utilizando el bisel de la aguja y el fiador curvo que posee el electrodo. Excepcionalmente será necesario abordar el espacio epidural desde el lado contrario al deseado para que el electrodo se aloje finalmente en el nivel y lado deseado. Una vez conseguidas las parestesias sobre la zona del dolor con la ayuda de un estimulador externo que es manipulado por un colaborador externo no estéril, se procede a tunelizar la conexión externa temporal. Con ella, se

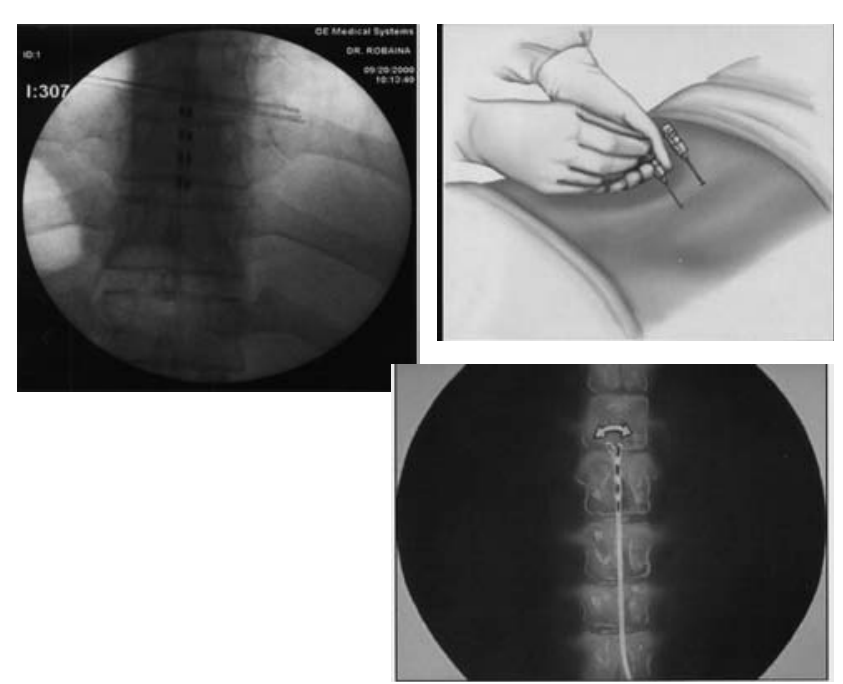

Figura 1. Arriba derecha: técnica de implante con doble punción. Arriba izquierda: dos electrodos paralelos implantados a nivel D9-D10. Abajo derecha: se muestra la posibilidad de desplazar lateralmente la punta del electrodo.
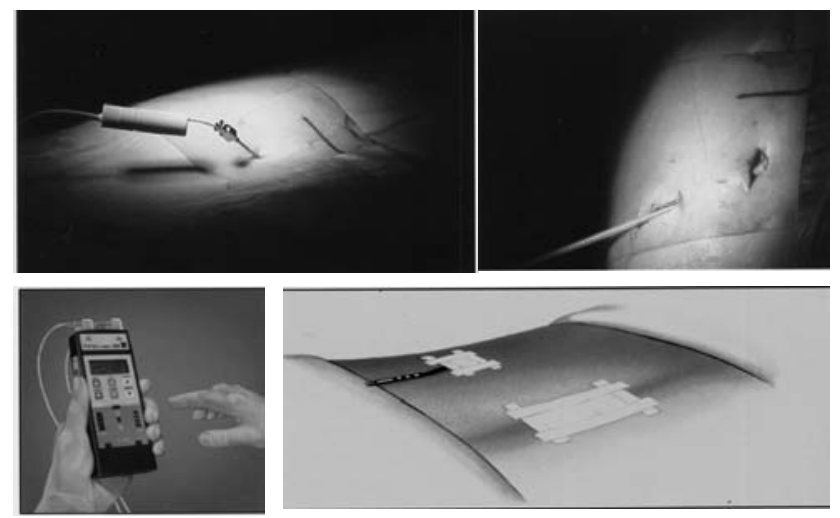

Figura 2. Arriba izquierda: extremo del electrodo conectado al cable para estimulación de prueba. Abajo izquierda: aparato de estimulación (testter) de prueba. Arriba derecha: tunelización de la conexión percutánea para la estimulación de prueba. Abajo derecha: conexiones externas percutáneas para fase de prueba.

procederá a realizar un programa de estimulación durante varias horas al día y en distintas situaciones de actividad física (subir y bajar escaleras, sentarse, levantarse, caminar, etc) durante una semana como mínimo (Figuras 1,2)

Si el resultado de la primera fase es favorable, en una segunda fase, se procede a la internalización del sistema, eliminando la conexión externa, implantando una nueva extensión subcutánea que se conecta a un neuroestimulador multiprogramable que se alojará en un bolsillo subcutáneo practicado en la pared abdominal, paraumbilical, generalmente en el lado izquierdo. A continuación, se procede a la programación del estimulador mediante un sistema de 

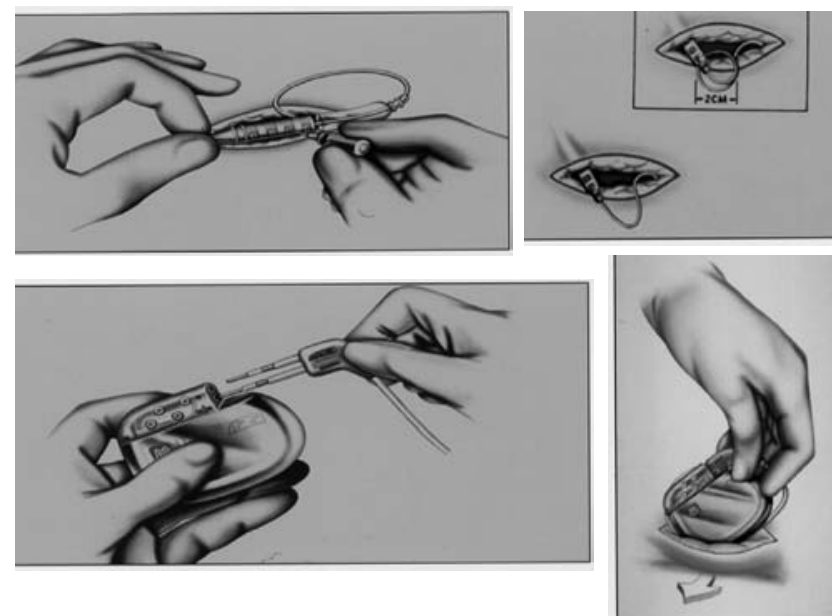

Figura 3. Se muestra los diferentes (de izquierda a derecha y de arriba abajo) pasos para la conexión definitiva de la extensión subcutánea al estimulador medular definitivo.

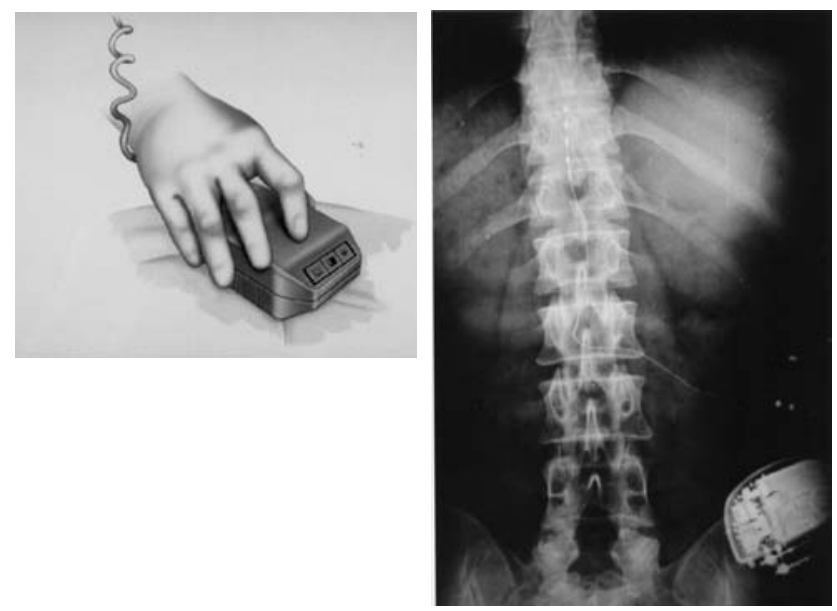

Figura 4. Izquierda: programación transcutánea mediante el cabezal del programador telemétrico. Derecha: imagen real del electrodo y estimulador implantados definitivamente. Obsérvese la laminectomía que existe en L4-L5 .

telemetría, programando los valores de intensidad de la corriente en voltios, frecuencia del estímulo en ciclos por segundo y la amplitud de onda en milisegundos. En general, los valores más frecuentes son : intensidad 4 voltios; frecuencia $90 \mathrm{~Hz}$ y amplitud de onda $200 \mu \mathrm{sec}$. El paciente dispone de un programador externo que le permite conectar y desconectar el sistema, así como modificar la intensidad de las parestesias al subir y bajar el voltaje de la corriente entre los límites previamente programados telemetricamente (Figuras 3,4)

Normalmente se implanta un electrodo tetrapolar, pero también es posible implantar dos electrodos tetrapolares paralelos o un electrodo octopolar con ocho puntos activos en línea e incluso dos octopolares paralelos. Actualmente, se encuentra en fase de estudio un comparativo entre los
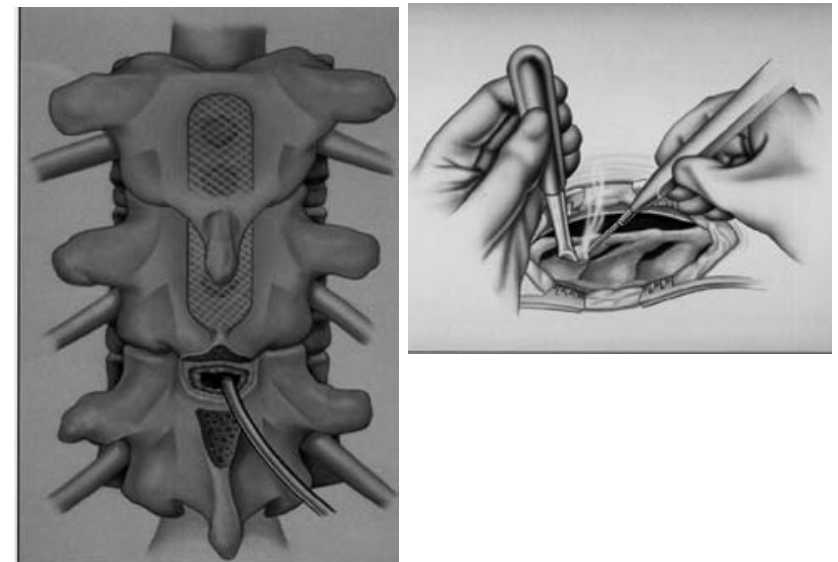

Figura 5. Dibujo que muestra la técnica de implante de un estimulador tetrapolar plano implantado a cielo abierto.
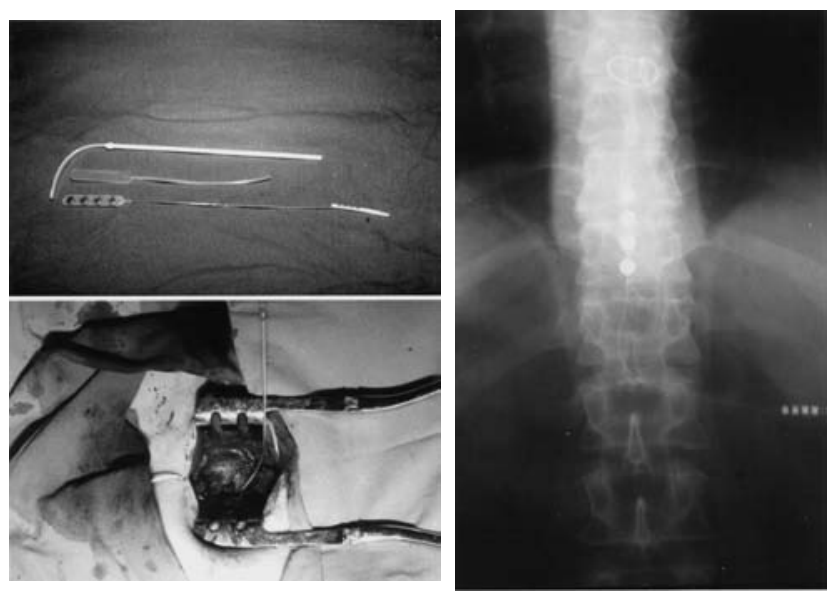

Figura 6. Imágenes reales de un implante de un electrodo tetrapolar plano implantado a cielo abierto. Arriba izquierda: Electrodo tetrapolar, simulador plástico y disector del espacio epidural.

resultados obtenidos con un simple electrodo cuadrapolar y los obtenidos implantando electrodos con múltiples polos activos o si existen variaciones favorables en el campo eléctrico que permitan aumentar el campo de percepción de las parestesias inducidas por la corriente eléctrica, con lo que aumentaría la posibilidad de tratar el dolor axial de la columna lumbar operada ${ }^{22}$.

En casos muy limitados bien por imposibilidad de progreso del electrodo en el espacio epidural por estenosis de canal, deformidades del raquis o desplazamiento del electrodo percutáneo, será preciso recurrir a la implantación de un electrodo plano cuadrapolar mediante una minilaminotomía a nivel D9-D10. El procedimiento puede realizarse con anestesia local o preferiblemente con anestesia general, aunque en este último caso se pierde la colaboración del paciente para la localización de las parestesias (Figuras 5, 6)

Las complicaciones derivadas de esta técnica son míni- 
Tabla II

Complicaciones de la neuroestimulación medular

\section{Derivadas de la técnica quirúrgica}

Infección de bolsillo subcutáneo y/o del trayecto de las conexiones

Meningitis

Fístulas de líquido cefalorraquídeo / cefalea postpunción

Hematoma epidural (1/2.000 implantes)

Parálisis transitoria postimplante sin causa aparente

Alteraciones de la sensibilidad- Alodinia
$\%$

5

0,5

1

0,05

1,8

4,2

\section{Complicaciones postimplante}

\subsection{Complicaciones de los equipos}

Migración del electrodo

Fractura del electrodo

Agotamiento de la batería antes de los 5 años

\subsection{Otras complicaciones}

Fenómeno de tolerancia a la sensación eléctrica

Decúbito sobre la zona de los implantes

Reacciones alérgicas locales

Precauciones al realizar estudios de RNM : parámetros a 0 y sistema apagado

mas y centradas fundamentalmente en fallos del sistema, desplazamientos y fracturas del electrodo e infecciones muy localizadas y superficiales a nivel de la zona de los implantes. Un estudio publicado recientemente en España, realiza una revisión exhaustiva de la bibliografía existente al respecto. Las complicaciones se dividen entre las derivadas de la técnica quirúrgica y las que se presentan postimplante. La Tabla II muestra las diferentes situaciones que pueden presentarse como complicaciones de la técnica ${ }^{5}$.

\section{Infusión espinal de medicamentos}

Otra técnica de neuromodulación muy efectiva en este tipo de pacientes consiste en la infusión espinal, generalmente intratecal, de morfina y otras sustancias con efectos analgésicos. Actualmente, aumenta progresivamente el número de pacientes portadores de bombas de infusión continua implantables y multiprogramables o de bombas de flujo estable. Los estudios de costo-beneficio han demostrado que los costos se igualan a los generados con los tratamientos farmacológicos y médicos en general a los seis meses del implante. Las indicaciones para un implante de una bomba de infusión deben hacerse tras un periodo de tratamiento con opiáceos orales $\mathrm{y}$, más recientemente, tras la utilización de parches dérmicos de opiáceos (buprenor- fina, fentanilo), siempre que aparezcan efectos secundarios o las dosis necesarias para controlar el dolor aumenten progresivamente. Otras sustancias que pueden administrase a nivel intratecal son los anestésicos locales (bupivacaína, lidocaína) y la clonidina ${ }^{11}$.

Un estudio reciente realizado en España, donde existe una experiencia muy elevada desde hace años con las bombas de infusión implantadas para el control de síndromes dolorosos complejos, hace mención muy clara a las indicaciones y a las evidencias clínicas de la aplicación terapéutica de la infusión espinal en el dolor crónico no oncológico. Uno de las premisas fundamentales básicas para aplicar esta técnica es que todos los tratamientos previos deben haber fallado ${ }^{21}$.

Para seleccionar el paciente para esta terapia es fundamental que éste comprenda perfectamente la técnica, sus ventajas e inconvenientes. Es preciso conocer la existencia de enfermedades intercurrentes que puedan hacer fracasar la técnica como son: la anemia aplásica, infección sistémica, alergias conocidas a metales o materiales de silicona o a los medicamentos que se van a infundir. La infusión espinal está totalmente contraindicada en caso de abuso activo de drogas por parte del paciente por el alto riesgo existente de contraer enfermedades sistémicas. Igualmente, existe contraindicación absoluta para el implante si presenta alte- 
raciones psiquiátricas mayores Las respuesta previa al uso de opioides sistémicos nos indicará si el paciente puede ser un candidato para esta técnica.

El paciente ideal es aquel que haya tenido una buena respuesta a los opiáceos por vía sistémica, cifrándose ésta en una disminución de la intensidad del dolor por lo menos el $50 \%$ del valor inicial, pero que ha presentado efectos adversos intolerables por esta ruta. Es preciso realizar varios test mediante la inyección intratecal de dosis crecientes de cloruro mórfico para valorar la respuesta. Existe la posibilidad de implantar un catéter subaracnoideo conectado a un sistema externo de infusión. En teoría la idea es buena pero en la práctica existen muchos problemas para conseguir una infusión estable, existiendo riesgo de infección además de complicar mucho las actividades de la vida diaria del paciente durante varias semanas ${ }^{36}$.

La forma más frecuente de realizar el test de prueba es mediante punciones lumbares cada $24-48$ horas con dosis crecientes en función de la respuesta al dolor y la aparición de efectos secundarios.

El fármaco más usado para el control del dolor lumbar y ciático postquirúrgico es la morfina, concretamente el cloruro mórfico sin conservante. Las dosis diarias oscilaran entre los $500 \mu \mathrm{gr} /$ día y los $24.000 \mu \mathrm{gr} /$ día. La concentración del fármaco condicionará la frecuencia de los rellenos periódicos de la bomba.

Pueden asociarse otros fármacos a la morfina con la finalidad de aliviar el componente neuropático en los miembros inferiores en este tipo de pacientes. Así, la bupivacaína que es un anestésico de larga duración puede combinarse con la morfina en dosis diarias generalmente alrededor de 2.400 $\mu \mathrm{gr} /$ día. La clonidina, un bloqueante alfa-adrenérgico, también se ha ensayado por vía intratecal en este tipo de dolor con resultados controvertidos. La hipotensión severa es el mayor riesgo que se corre al emplear este fármaco.

En el mercado existen diferentes sistemas de infusión continua implantables, dividiéndose básicamente en sistemas de flujo constante y sistemas de flujo variable. Un ejemplo de bomba de flujo constante es la Infusaid (Infusaid, Inc,Nordwood, Mass, USA) y de flujo variable y multiprogramable la SynchroMed (Medtronic, Inc; Minneapolis, USA) ${ }^{7}$.

La bomba de flujo constante (Infusaid)) funciona mediante un líquido propelente que se transforma en gas con los rellenos y vaciados respectivamente, siendo por lo tanto una fuente de energía inagotable. Es de titanio y permite el relleno percutáneo a través de un acceso central del silicona. El volumen del reservorio es de $50 \mathrm{ml}$. La velocidad de vaciado puede verse afectada con los cambios de altitud hasta un $45 \%$ de incremento de flujo. Incluso los vuelos comerciales presurizados pueden afectarla. Igualmente, un aumento de la temperatura corporal por encima de los $37^{\circ} \mathrm{C}$ puede resultar en un aumento del flujo de la bomba entre un $10-13 \%$ por cada ${ }^{\circ} \mathrm{C}$ de incremento. La hipotermia produce el efecto contrario.

La bomba de flujo variable (Syncromed) es multiprogramable y diseñada para la administración de medicación en diferentes lugares del cuerpo. El sistema se compone de una bomba de titanio y un programador externo que controla y programa la bomba implantada. Contiene un motor que funciona por pulsos emitidos por una batería interna. La duración de la batería está en función de la cantidad de flujo, oscilando entre 3 a 5 años. El reservorio es de 18 $\mathrm{ml}$. Una minibomba peristáltica extrae el contenido del reservorio a través de un microfiltro antibacteriano impulsándolo por el sistema de catéteres.

La Synmcromed está equipada con varias medidas de seguridad, un sistema de alarma de volumen y de baja batería y un filtro antibacteriano de $0.22 \mu \mathrm{m}$ entre el reservorio y la microbomba peristáltica. Este filtro es muy importante ya que impide el paso al espacio subaracnoideo de sustancias y bacterias que podrían originar una meningitis.

Una antena permite la comunicación entre el programador externo y la bomba mediante telemetría con radiofrecuencia. Es posible conocer por esta vía datos sobre la medicación, concentración, volumen residual, dosis diaria, así como la programación de distintos programas de infusión

Las preferencias actuales de la mayoría de las unidades del dolor españolas es implantar en el dolor lumbar y ciático postquirúrgico una bomba multiprogramable. Quizá, en aquellos casos en los que exista estabilidad clínica y de la dosis a lo largo de varios años, en el momento del reemplazo por agotamiento de la batería de una bomba multiprogramable, se contemplaría implantar una bomba de flujo constante.

\section{Técnica implante de una bomba de infusión}

Después de realizar los test de prueba para conocer la respuesta al tratamiento, el implante del sistema debe hacerse en quirófano, con las máximas condiciones de asepsia y empleando profilaxis antibiótica antes y durante el procedimiento.

La técnica de implante es básicamente la misma para ambos tipos de bombas. El paciente se coloca en decúbito lateral con el flanco donde se implantará la bomba en la parte superior. De esta manera se tienen acceso a la región lumbar y abdominal. Esta misma posición se adoptará en caso de revisiones del catéter o de la bomba una vez implantados los mismos si existen problemas con la infusión o se va a proceder al reemplazo de la bomba o alguno de los catéteres.

La anestesia durante el implante estará condicionada por una serie de factores entre los que destacan los antecedentes clínicos, la edad, alteraciones físicas y las preferencias 

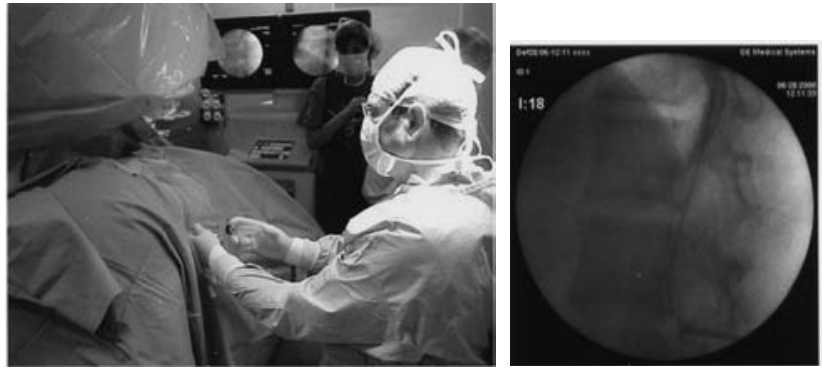

Figura 7. Izquierda: técnica de implante del catéter subaracnoideo. Máximas condiciones de asepsia. Paciente en decúbito lateral. Derecha: Obsérvese el ascenso del catéter (con fiador) en el espacio subaracnoideo lumbar.

del cirujano y del paciente. En general una anestesia local de la zona de punción, de la zona del implante de la bomba y del trayecto de los catéteres, suele ser suficiente. La administración de algún hipnótico y opiáceo en dosis bajas por vía intravenosa contribuye al confort del paciente a lo largo del procedimiento (Midazolam, Propofol, Fentanilo, Sufentanilo).

El catéter se implanta siempre en el espacio subaracnoideo mediante una punción lumbar con una aguja de Tuhoy a nivel L3-L4. Una vez obtenido el líquido cefalorraquídeo (LCR), el catéter primario se hace ascender bajo control fluoroscópico (Figura 7). Este aspecto de la técnica es muy importante puesto que evita la emigración caudal del catéter, así como los posibles bucles y acodamientos del mismo. En ocasiones, estos acodamientos, pueden originar dolor persistente por irritación radicular u obstrucción del flujo de LCR a través del catéter. El extremo distal del catéter se suele localizar a nivel D12-L1, aunque en este punto no existe acuerdo, pudiéndose si se desea ascender algún nivel más. Solamente la cuarta parte de la medicación infundida a nivel lumbar puede alcanzar niveles cerebrales.

Una vez que se ha colocado la punta del catéter en el nivel deseado, se procede entonces a retirar el fiador metálico del interior del catéter y posteriormente se retira la aguja, se tuneliza el catéter hasta el costado del paciente donde se conecta subcutáneamente con el extremo distal del catéter secundario que viene de la bomba la cual se encuentra alojada en un bolsillo subcutáneo a nivel del flanco. Antes del implante, la bomba debe rellenarse de la solución de morfina. Posteriormente, una vez ya implantada la bomba subcutáneamente, se procederá a la programación de la misma mediante telemetría con un programador externo (Figura 8).

\section{Complicaciones postoperatorias de las bombas de infu- sión}

Las complicaciones pueden presentarse como conse-
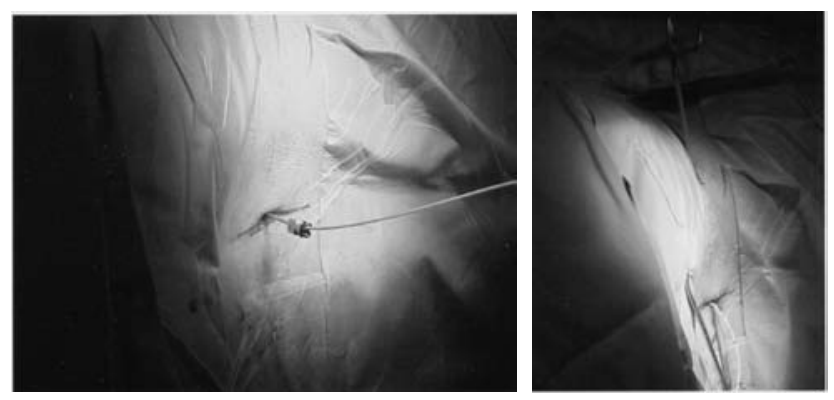

Figura 8: Izquierda a derecha y arriba abajo: Catéter externalizado, tunelizado y conectado a la bomba de infusión multiprogramable antes del implante definitivo en la pared abdominal

cuencia del implante del catéter o de la bomba. No suelen ser muy frecuentes en los sistemas totalmente implantados.

Después del implante del catéter puede presentarse inmediatamente sangrado profuso. Más tardíamente podría presentarse cefalea, fístula de LCR, infección, malfunción del catéter y crisis vegetativas.

El sangrado después de la punción o de la tunelización es frecuente, aunque no suele representar un problema serio. Sí podría serlo si el paciente presenta previamente alteraciones no conocidas de la coagulación. El hematoma epidural es poco frecuente pero representa una complicación muy grave. Su incidencia se cifra en 1 caso por cada 220.000 procedimientos espinales. Puede deberse a la punción directa de una vena epidural y/o a una rotura o canalización de una vena epidural por el catéter. La clínica de esta complicación se presentará varias horas después del implante en forma de dolor lumbar intenso y progresivo, seguido de sensación de debilidad en los miembros inferiores, pudiendo evolucionar hacia un síndrome florido de compresión medular y/o de la cola de caballo en poco tiempo. El diagnóstico clínico debe confirmarse mediante una tomografía axial computarizada ((TAC) o una resonancia nuclear magnética (RNM). La solución es claramente quirúrgica mediante la realización de una laminectomía descompresiva.

Infección Subcutánea. Puede presentarse en el punto de punción lumbar, en el trayecto o en la zona del implante de la bomba. Esta complicación implica casi invariablemente la necesidad de explantar de todo el sistema. Es posible que si se autolimita la infección con el correspondiente tratamiento antibiótico, pudieran conservarse algunos de los elementos implantados. El estafilococo aureus suele ser el germen más frecuentemente encontrado en los cultivos.

Absceso epidural. Es una posibilidad a tener en cuenta aunque su presentación ha sido meramente anecdótica. El tratamiento sería exclusivamente quirúrgico.

Meningitis bacteriana. Esta complicación es poco fre- 
cuente. Ante la mínima sospecha de su existencia se debe explantar todo el sistema. Debe identificarse el germen causante de la infección e iniciar la correspondiente terapia antibiótica intravenosa.

La mayor parte de los problemas derivados de los sistemas totalmente implantados se presentan con los catéteres, afectando hasta un $34 \%$ de los pacientes. Los más frecuentes son la emigración, anudamiento, rotura y oclusión.

La formación de un granuloma de cuerpo extraño en la punta del catéter intratecal es una complicación poco frecuente, pero que debe tenerse en cuenta porque terminará por obstruirlo. En estas situaciones se produce un descenso progresivo en la eficacia del sistema. Una RNM podría poner de manifiesto la existencia del problema, obligando en algunos casos a la extirpación quirúrgica del catéter.

Las bombas multiprogramables de flujo variable, al tener una batería interna se agotan con el paso de los años, debiéndose reemplazar por una nueva cada 3 a 5 años. El sistema de catéteres puede en muchos casos, permanecer intacto.

La fiabilidad mecánica de las bombas multiprogramables actuales es muy elevada, hasta el punto que pueden descartarse fallos mecánicos casi por completo. Son mucho más probables los fallos humanos durante la programación de la bomba ${ }^{8}$.

\section{Complicaciones derivadas de la infusión de fármacos}

Como el fármaco de elección para el tratamiento del dolor lumbar y la ciática postquirúrgica es fundamentalmente con opiáceos intratecales, nos referiremos exclusivamente a los efectos secundarios de la infusión de morfina. Estos, pueden presentarse de forma aguda o crónica. Entre los primeros destacan el prurito, náuseas, vómitos, retención urinaria y depresión respiratoria. Entre los segundos destacan por orden de frecuencia de presentación el estreñimiento, somnolencia, trastornos cognitivos, sudoración profusa, ganancia o pérdida de peso, disminución de la líbido, amenorrea, retención de líquidos con edemas de miembros inferiores. Otros efectos secundarios pero menos frecuentes serían recurrencias de infecciones herpéticas labiales, disfunciones oculares, vértigo y disfunciones termorreguladoras.

En aquellos casos de dolor rebelde persistente e incapacitante en pacientes intervenidos de la columna lumbar, en los que han fracasado todas las estrategias mínimamente invasivas e incluso la reintervención quirúrgica en forma de artrodesis intersomáticas y o transpediculares, la técnica de infusión intratecal de morfina se presenta como una alternativa muy válida y de la que se dispone de una gran experiencia en nuestro país.

\section{Conclusiones}

Como hemos visto, las técnicas de neuromodulación medular tanto mediante estimulación eléctrica como la modulación farmacológica, aplicadas al manejo del dolor postcirugía discal y lumbar en general, representan una alternativa válida e incluso mejor en la gran mayoría de casos en comparación con la nueva cirugía o la artrodesis instrumentada. Está última técnica, como veremos en otro artículo remitido para su publicación en esta misma revista, no soporta los criterios de la medicina basada en la evidencia, por lo que salvo que exista una manifiesta inestabilidad o compresión radicular, no debería aplicarse cirugía, sino recurrir a las técnicas intervencionistas de manejo del dolor crónico lumbar y finalmente aplicar las técnicas de neuromodulación descritas en este artículo, cuyos resultados clínicos y de costo-beneficio son más favorables, como se ha podido demostrar por las revisiones bibliográficas aportadas.

Recordar finalmente, que los neurocirujanos estamos llamados nuevamente a participar activamente en el campo del alivio del dolor crónico mediante la aplicación de las nuevas técnicas de neuromodulación tanto a nivel medular y cerebral. Este punto enlaza con el gran campo que la neuromodulación terapéutica abre también actualmente y nuevamente a la neurocirugía funcional, como es el papel primordial que va a jugar la neurocirugía en el manejo de los movimientos anormales, la psicocirugía y el restablecimiento de las funciones neurológicas perdidas.

\section{Bibliografía}

1. Baeza, J.: Infiltración neuroaxial con ozono. Fisiopatología y mecanismos de acción. Rev. Soc. Esp. Dolor 2005; 12: Extra.II: 18-23.

2. Bocci, V.: Mecanismos de acción generales de la ozonoterapia y mecanismos en el tratamiento del dolor. Rev. Soc. Esp. Dolor 2005; 12: Extra II: 24-36.

3. Bono, C., Lee, C.: Critical analisis of trens in fusion for degenerative disc disease over the past 20 years: Influence of technique of fusion rate and clinical outcome Spine 2004; 29 : 455-463.

4. Brox, J., Reikeras, O., Nygaard, O., et al.: Lumbar instrumented fusion compared with cognitive intervention and exercises in patients with chronic back pain after previous surgery for disc herniation. A prospective randomized controlled study. Pain 2006; 122: 145-155.

5. Cerdá-Olmedo, G., de Andrés, J.: Complicaciones de la neuroestimulación medular. Rev. Soc. Esp. Dolor 2003; 10: Supl. I: 126-136.

6. Clavo, B., Robaina, F., Gutiérrez, D., et al.: Historia y experiencia con ozonoterapia en el Hospital Universitario Dr. Negrín de Las Palmas. Rev. Soc. Esp.Dolor 2005; 12: Extra II: 
53-60.

7 Gianino, J.M.; York, M.M., Paice, J.A.: Intrathecal drug therapy for spasticity and pain. Springer. 1995.

8. González-Escala, J.R.: Complicaciones de la infusión espinal. Rev. Soc. Esp. Dolor 2003; 10: Supl. I: 55-76.

9. Karpinen, J.: Tumor necrosis factor-alfa monoclonal antibody, inflimax, used to management severe ciatica. Spine 2003; 28 : 750-753.

10. Krames, E.: The right place at the right time. Neuromodulation 2005; 8: 153-154.

11. Krames, E.: Intrathecal infusional therapies for intractable pain. Patient management guidelines. J Pain Symptom Management 1993: 8-36.

12. Kumar, K., Malo, K.S., Demeria, D.: Treatment of chronic pain with spinal cord stimulation versus alternative therapies: cost-effectiveness analisis. Neurosurgery 2002; 51 : 106-115.

13. Melzac, R., Wall, P.D.: Pain Mechanism:a new theory. Science 1965; 150: 971-978.

14. Murga, M.: Infiltración neuroaxial con ozono. Indicaciones, técnicas y experiencia clínica. Rev. Soc. Esp. Dolor 2005; 12: Extra II: 10-17.

15. North, R.B., Wetzel, F.T.: Spinal cord stimulation for chronic pain of spinal origin: a valuable long-term solution. Spine 2002; 27: 2584-2591.

16. Polomano, R., Marcotte, P.: Spinal fusion or exercise and cognitive intervention? In search of the answers. Pain 2006; 122: 4-5.

17. Robaina, F.; Manejo intervencionista de la ciática y del dolor lumbar postquirúrgico. Rev. Soc. Esp. Dolor 1999; 6: 233-237.

18. Robaina, F.: Situación actual de la ozonoterapia. Rev. Soc, Esp. Dolor 2005; 12; Extra II: 1-2.
19. Robaina Padrón, F.J.: Aspectos neuroquirúrgicos del tratamiento del dolor crónico en el tercer milenio. Las unidades multidisciplinarias del dolor. Rev. Soc. Esp. Dolor 2003; 10: 481-507.

20. Robaina, F.: Situación actual de la cirugía de la columna vertebral degenerativa aplicada al manejo del dolor lumbar crónico. Estenosis de canal. Discopatía degenerativa, resultados basados en la evidencia científica. Rev. Soc. Esp. Dolor 2006; 3: 167-172.

21. Rodríguez, M., Aldaya, C., de la Torre, R.: Indicaciones y evidencias clínicas en la aplicación terapéutica de la infusión espinal. Rev. Soc. Esp. Dolor 2003; 10: Supl. I: 19-32.

22. Van Buyten, J.: Tratamiento del dolor crónico de espalda y miembro inferior; avances en la aplicación de la estimulación de la médula espinal. Rev. Soc. Esp. Dolor 2003; 10; Supl. I: 116-125.

23. ViK, A., Zwart, J., Hulleberg, G., et al.: Eight year outcome after surgery for lumbar disc herniation: a comparison of reoperated and not operated patients. Acta Neurochir 2001; 143: 607-610.

24. Wiesel, S.: Changing evidence Standard undermine the artificial disc. The Back Letter 2005; 20: 73-78.

Robaina Padrón, F.J.: Sindrome postlaminectomía lumbar II. Tratamiento del dolor mediante técnicas de neuromodulación. Neurocirugía 2008; 19: 35-44.

Correspondencia postal: Dr. Francisco Javier Robaina Padrón. Jefe de la Unidad del Dolor Crónico y Neurocirugía Funcional. Unidad del Dolor Crónico y Neurocirugía Funcional. Nivel 5. Hospital Universitario de Gran Canaria "Dr. Negrín”. C) Barranco de la Ballena s/n. 35010 Las Palmas de Gran Canaria. 